\title{
EVALUATION OF DAYLIGHT AND THERMAL INFRA-RED BASED DETECTION FOR PLATOONING VEHICLES
}

\author{
WOEBER, W[ilfried]; KEFER, M[artin]; KUBINGER, W[ilfried] \& SZUEGYI, D[aniel]
}

\begin{abstract}
The research project "SafeCon" tackles the problem of the safety in military transport missions. In such missions people can be killed by attacks or natural dangers, for example avalanches. To reduce the number of involved people in transport missions, SafeCon tries to reduce the number of requied personnel by automating each transporter.The aim of SafeCon is the realisation of a semi-autonomous truck convoy. The destruction of one vehicle within the convoy causes financial losses and spares human lives. Further, the vehicles must not be armoured. This reduces both load capacity and costs.

For an autonomous behaviour, each truck must be able to follow the vehicle ahead. Because of that, each vehicle must be able to recognise the leading vehicle. This is realised with a computer visiondetection system. To enable an autonomous behaviour in all weather conditions a thermal infra-red camera system is implemented to detect objects based on their heat signature. Different to other approaches, this paper discusses the implementation of an AdaBoost based detection for thermal infra-red image material.

The evaluation of our approach compares a thermal infra-red and a visible-light detection. Ourexperiments prove that the thermal infra-red detection is comparable with visible-light detection.

Keywords: Thermalinfra-red,Object detection, AdaBoost, Autonomous vehicle
\end{abstract}

\section{MOTIVATION}

SafeCon - Safe semi-autonomous convoyingtackles the problem of safety in military transport missions. These missions can take place in dangerous areaswhere human casualties due to enemy attacks or natural catastrophes are a factor. To decrease the number of human participants, SafeCon develops an autonomous vehicle convoy. Each vehicle is equipped with a number of sensors, actuators and control systems. In cooperation of all electronic, mechatronic and mechanic parts, an autonomous behaviour is realised. But for the overall movement of the convoy, each vehicle must be able to follow the vehicle ahead in all weather conditions[1], [2],
[8]. Therefore, a thermal infra-red based computer vision system was implemented to analyse heat differences in the vehicles environment.

Previous approaches detect pedestrians by analysing the heat signature (see [12] and [13]). Another approachcomparesa stereo camerafor visible light images and thermal images [11]. Reference [14] discusses a face detection approach based on heat signature. The detection process is based on the unique vein structure and minutiae extraction.

This paper discusses the implementation of a machine learning process and compares a visible light and a thermal infra-red AdaBoost detection application. The aim of this work is an evaluation of the efficiency of a thermal infra-red based AdaBoost object detection.

The paper is organised as follows. Section 2 describes the system concept. The detection approaches are explained in section 3. Section 4 shows the experimental results andsection 5 summarises the paper.

\section{SYSTEM CONCEPT}

The main part of the sensing equipment is a sensor rack which is mounted on the top of the vehicles cockpit. Fig. 1shows the sensor rack.

The rack is based on a solid rig made of Robotunits rods (4). All optical sensors are mounted on this rig. The Basler avA1600-50gm RGB camera and the Basler avA1600-50gc greyscale cameras (1) are used to employ the environment by capturing visible light. The Velodyne HDL-32E laser scanner (2) model the environment by multiple distance measurements. For the all-weatherdetection two FLIR PathFindIR thermal infra-red cameras (3) are implemented.

As mentioned before, all vehicles within the convoy must be able to follow the vehicle ahead. Therefore, an

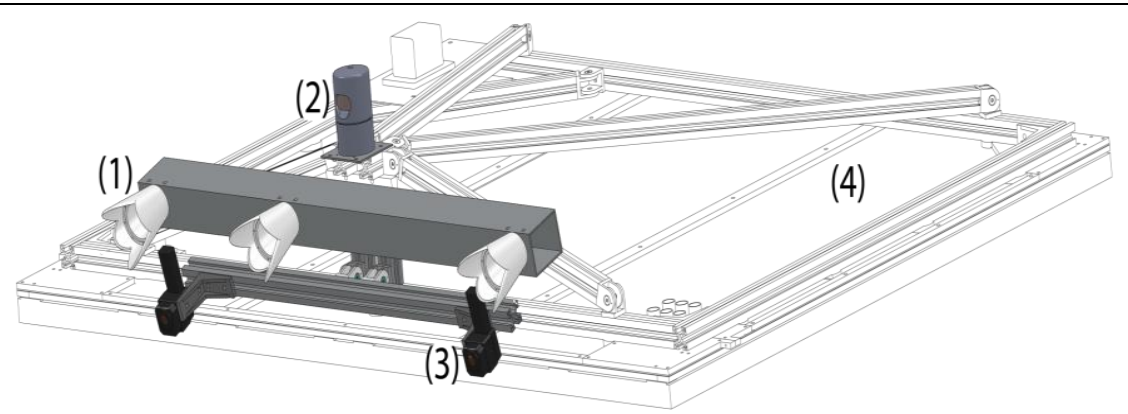

Fig. 1. System concept 


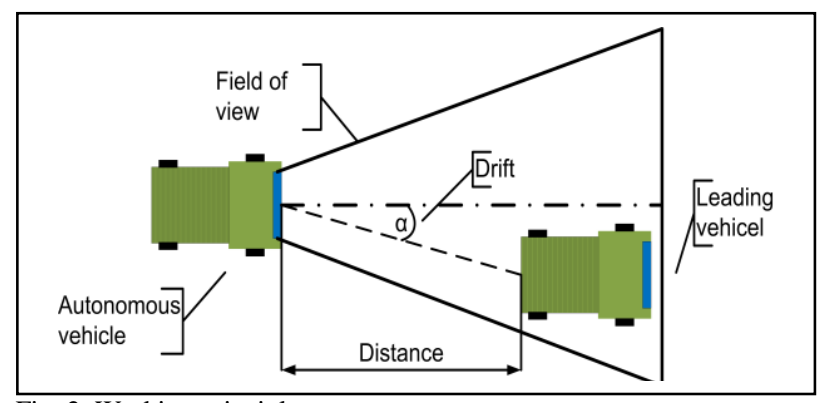

Fig. 2. Working principle

AdaBoost based computer vision system (CVS) is implemented.The main part of our work is the implementation of an existing AdaBoost algorithm in our CVS and the development of an AdaBoost learning procedure. Further, evaluation software for the detection process was developed.

The CVS analyses the image material and searches for the truck ahead. Based on this image analysisthe drift and distance to the leading vehicle can be calculated. Fig. 2 shows the described working principle. The CVS uses a digital description of the vehicle for the detection process which is calculated in a learning process. The digital object description is called classifier. The CVS and the generation of the classifier are described in the following sections.

\section{COMPUTER VISION DETECTION}

For the detection process, an AdaBoost based approach was chosen. AdaBoost is a machine learning approach which is described in [2] and used in [5] and [6]. This machine learning algorithm tries to find regularities in input material, in this case image material. The result of the AdaBoost learning process is a digital object description called classifier.

All used algorithms are based on the OpenCV 2.1 library [3],[9].

\subsection{AdaBoost}

The final classifier is based on hypothesis which tries to recognise the needed information in the input material. The idea of the AdaBoost learning process is the weighted combination of "weak" hypothesis. Each weak hypothesis performs a little bit better than random. The learning process calculates the contribution of each weak hypothesis to the final hypothesis, the classifier [2].

In this case, the input material is the image material. For an accurate classifier, the input material must cover all possible positions of the truck. This leads to a huge number of input images. This image material is analysed with image features which describes the image content. The result of the image analysis is the base of the learning process [3], [9].

The input images are divided into positive and negative images. The positive images show the truck and the negative images everything else (e.g. background). For an accurate learning process, the position of the truck must be marked within the positive image material. To automate this process a template matching algorithm is implemented. This approach enables an almost fully automated learning process.

\subsection{Template matching}

The template matching algorithm is used to mark the position of the truck in the positive image material. The template matching algorithm is described in [7]. Simply put, an example image of the truck is stored as a template which is used to find the truck in other images.

To find the truck in an image the template slides over each image. On each position the difference between the template and the current position within the image is calculated. If the difference is below a certain threshold, a match is detected. A perfect match will cause no difference between the template and the image. In this case, the difference is calculated with the "normalised square difference method" of OpenCV. See [3] and [9]for more details. Equation (1) shows that calculation [3].

$$
\begin{aligned}
& D_{\text {normed }}=\frac{D_{D i f f}(x, y)}{Z(x, y)}= \\
& =\frac{\sum_{x^{\prime}, y_{\prime}}\left[T\left(x^{\prime}, y^{\prime}\right)-I\left(x+x^{\prime}, y+y^{\prime}\right)\right]^{2}}{\sqrt{\sum_{x^{\prime}, y^{\prime}} T\left(x^{\prime}, y^{\prime}\right)^{2} \cdot \sum_{x^{\prime}, y^{\prime}} I\left(x+x^{\prime}, y+y^{\prime}\right)^{2}}}
\end{aligned}
$$

Where $T$ is the template image and $I$ is the positive input image. $Z$ describes the normalizations coefficient. When $D_{\text {normed }}$ is below a threshold value, a match is found. The coordinates $x$ and $y$ describes the position in the input image and the coordinates $x^{\prime}$ and $y^{\prime}$ the position in the template image.

Basically the template matching algorithm can be used in the CVS for the detection process. It is very fast and simple. But the template matching algorithm must be initialised manually. Further, because of the template, just a single view of the truck can be detected. Drifts and changes in distance cause the algorithm to fail.

The AdaBoost approach is much more robust against changes in perspective. Further, the AdaBoost approach performs fast enough for this application.

\subsection{Image material}

The image material was taken from measurement runs. The aim of those runswas to capture the vehicle in many different positions, angels and distances. The sensor rack was mounted on a car. All calculated classifiers and test scenarios are based on the measurementruns. To compare both, the thermal infrared detection and the visible light detection, the visible light images were shrinked. That is necessary because of the different number of pixel. Tab. 1 shows the size of the image material.

\begin{tabular}{|c|c|c|c|}
\hline & \multicolumn{3}{|c|}{ Image size } \\
\cline { 2 - 4 } & Width & Height & Nr. Of Pixel \\
\hline Thermal infra-red & 320 & 240 & 76800 \\
\hline $\begin{array}{c}\text { Visible light } \\
\text { (original) }\end{array}$ & 1600 & 700 & 1120000 \\
\hline $\begin{array}{c}\text { Visible light } \\
\text { (shrinked) }\end{array}$ & 419 & 183 & 76600 \\
\hline
\end{tabular}

Tab. 1. Image size

The shrinking process was realised by the method "cvResize" of the OpenCV library. See [3] and [9] for 
more information.Fig. 3shows the vehicle displayed in visible light and thermalinfra-red. The red rectangle frames the vehicle.

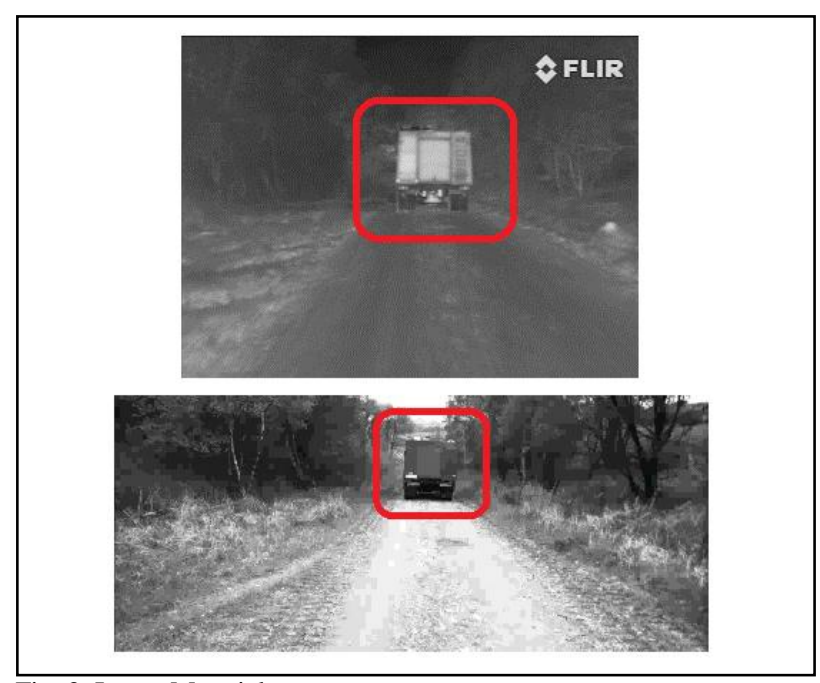

Fig. 3. Image Material

\subsection{Learning Method}

As mentioned before, the learning process is based on the positive image material prepared by the templatematching algorithm. This section gives detailed information of the learning process of the system and the connection between the template matching and the AdaBoost learning algorithm.

First, all positive images are stored in AVI files. From all video files, a template is extracted manually and stored in a template library. These templates are used to extract the position of the truck in all images in a video file. If the template matching algorithm fails, the template must be updated by hand. The prepared image material is used to calculate the AdaBoost classifier.Fig. 4shows the learning algorithm.

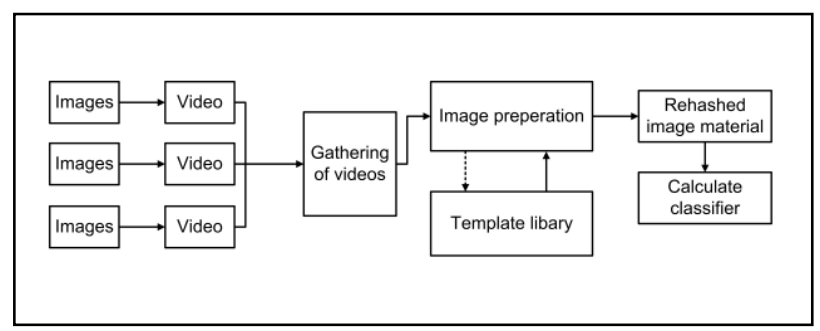

Fig. 4. Learning algorithm

For the comparison ofvisible light and thermal infra-red detection different classifier were calculated. Because of the huge amount of details in visible light images, the learning process for visible light images takes much more time and exhibits more errors in the preparation process. That means that the template must be updated frequently. Because of the small image size and less details in thermal infra-red images, the learning process is almostfully automated.

\section{EXPERIMENTS}

The aim of this paper is to evaluate the thermal infra-red objet detection. To prove the quality of our approach, the thermal infra-red detection was compared with visible light detection.
To show the impact of the quantity of input image material, multiple classifiers were calculated. This section explains the experimental process and evaluates the thermal infra-red approach.

\subsection{Classifiers}

As mentioned in section 3, the number of images and the quality are determining for the detection result.Tab. 2 and Tab. 3 shows the details for thermal infra-red (TIR) and visible light (VIS) classifiers.

\begin{tabular}{|l|l|l|l|}
\hline \multirow{2}{*}{ Source } & \multicolumn{3}{|c|}{ Thermal infra-red classifier } \\
\cline { 2 - 4 } & \multicolumn{1}{|c|}{ Name } & \multicolumn{1}{c|}{ Nr. Images } & \multicolumn{1}{c|}{$\begin{array}{c}\text { Image } \\
\text { quality }\end{array}$} \\
\hline TIR & C1-IR & about 1700 & Bad \\
\hline TIR & C2-IR & about 3600 & Bad \\
\hline TIR & C3-IR & about 100 & Good \\
\hline TIR & C4-IR & about 500 & Bad \\
\hline TIR & C5-IR & about 1000 & Good \\
\hline TIR & C6-IR & about 3000 & Good \\
\hline
\end{tabular}

Tab. 2. Thermal infra-red Classifier

\begin{tabular}{|l|l|l|l|}
\hline \multirow{2}{*}{ Source } & \multicolumn{3}{|c|}{ Visible light classifier } \\
\cline { 2 - 4 } & \multicolumn{1}{|c|}{ Name } & \multicolumn{1}{|c|}{ Nr. Images } & \multicolumn{1}{c|}{$\begin{array}{c}\text { Image } \\
\text { quality }\end{array}$} \\
\hline VIS & C1-VIS & about 310 & Good \\
\hline VIS & C2-VIS & about 510 & Good \\
\hline VIS & C3-VIS & about 760 & Good \\
\hline
\end{tabular}

Tab. 3. Visible light classifier

\subsection{Experimental results}

This section shows the results of the vehicle detection. As mentioned before, the results are based on different scenarios from measurement drives. Tab. 4 shows the different scenarios.

\begin{tabular}{|l|l|l|}
\hline \multirow{2}{*}{ Source } & \multicolumn{2}{c|}{ Scenarios } \\
\cline { 2 - 3 } TIR & \multicolumn{1}{|c|}{ Name } & \multicolumn{1}{c|}{ Nr. Images } \\
\hline TIR & Scenario 1 & 2000 \\
\hline TIR & Scenario 2 & 4000 \\
\hline VIS & Scenario 1 & 20388 \\
\hline VIS & Scenario 2 & 3505 \\
\hline VIS & Scenario 3 & 6966 \\
\hline VIS & Scenario 4 & 10383 \\
\hline
\end{tabular}

Tab. 4. Scenarios

The error rates of the detection process were measured manually. All experiments were performed with the same settings of the detection algorithm. Reference [3] and [9]describes the algorithm more detailed.

Tab. 5 shows the result for classifier C1-IR, C3-IR, C4-IR and C5-IR. The matching rate in Tab. 5features an error rate less than $0,7 \%$. The results of the classifiers C2-IR and C6-IR are shown in Tab. 6. The result is based on an error rate from $2,6 \%$ and $6 \%$. 


\begin{tabular}{|c|c|c|c|}
\hline \multirow{2}{*}{ Source } & \multicolumn{3}{|c|}{ Result of detection process } \\
\cline { 2 - 4 } & Scen. I & Scen. II & Scen. III \\
\hline C1-IR & $94,8 \%$ & $97,4 \%$ & $78,8 \%$ \\
\hline C3-IR & $92,1 \%$ & $65,15 \%$ & $51.0 \%$ \\
\hline C4-IR & $86,6 \%$ & $47,42 \%$ & $29,0 \%$ \\
\hline C5-IR & $97,8 \%$ & $98,9 \%$ & $93,17 \%$ \\
\hline
\end{tabular}

Tab. 5. Result TIR I

\begin{tabular}{|c|c|c|c|}
\hline \multirow{2}{*}{ Source } & \multicolumn{3}{|c|}{ Result of detection process } \\
\cline { 2 - 4 } & Scen. I & Scen. II & Scen. III \\
\hline C2-IR & $98,5 \%$ & $99,2 \%$ & $94,7 \%$ \\
\hline C6-IR & $98,9 \%$ & $99,4 \%$ & $96,2 \%$ \\
\hline
\end{tabular}

Tab. 6. Result TIR II

To decrease the error rate of the classifiers C2-IR and C6-IR, the threshold of the OpenCV detection algorithm was changed. This Threshold is called "minNeighbors".

This value controls the decision making of the detection algorithm. For more information see [3] and [9]. The result is a lower matching rate (about 92,3\%) but an error rate under $0,1 \%$.Our results show that the classifiers with better quality exhibit better matching rates.

The results for the calculated visible light classifiers are shown in Tab. 7.

\begin{tabular}{|c|c|c|c|c|}
\hline \multirow{2}{*}{ Source } & \multicolumn{4}{|c|}{ Result of detection process } \\
\cline { 2 - 5 } & Scen. I & Scen. II & Scen. VI & Scen. III \\
\hline C1-VIS & $33,9 \%$ & $27,7 \%$ & $41,0 \%$ & $51,15 \%$ \\
\hline C2-VIS & $98,1 \%$ & $95,5 \%$ & $95,2 \%$ & $96,6 \%$ \\
\hline C3-VIS & $98,9 \%$ & $99,4 \%$ & $97,3 \%$ & $97,9 \%$ \\
\hline
\end{tabular}

Tab. 7. Results VIS

Additional to the listed scenarios, another scenario was tested. This scenario is similar to the scenario III of the thermal infra-red scenarios. The result of this scenario was a matching rate about $81,9 \%$ and an error rate of $3,7 \%$. This low matching rate occurs because of shadowy areas within the scenario and the low number of positive images material of the learning process.

The experiments show that the developed thermal infra-red approach cannot reach the accuracy of the visible light detection. To increase the performance of the thermal infra-red detection, the quality and size of the image material must be enhanced. However, the big advantage of the developed detection system is the allweather applicability.

\section{SUMMARY AND CONCLUSION}

This paper presents an evaluation for thermal infrared object detection. The theoretical background of both the AdaBoost approach and the template matching approach were discussed shortly.

The AdaBoost learning process is supported by the template matching image preparation.For the evaluation multiple classifiers for thermal infra-red and visible light were calculated. Thermal infra-red classifiers and visible light classifiers were compared and evaluated. Both approaches perform very well.
However, the accuracy of the visible light classifiers cannot be reached by thermal infra-red classifiers. But because of the good performance of the thermal infra-red classifier under all weather conditions, the performance of the thermal infra-red classifier is acceptable.

The next step is the calculation of the position of the vehicle ahead based on the detection results and the implementation of a filter system (for example a Kalman filter [10]) to realise object tracking.

The calculated position of the vehicle ahead will be used to manipulate the vehicles navigation. The movement will be aligned to the vehicle ahead. That leads to a smoother motion of the convoy.

\section{ACKNOWLEDEMENT}

This project has been funded by the Austrian Security Research Programme KIRAS - an initiative of the Austrian Federal Ministry for Transport, Innovation and Technology (bmvit).

\section{REFERENCES}

[1] Huang, Q., Benmimoun, A., Robinson, T. \& Bergenheim, C. (2010).,,Challenges of Platooning on Public Motorways". In: $17^{\text {th }}$ World Congress on Intelligent Transport Systems

[2] Schapire, R.E.. \& Freud Y. (1999). "A Short Introduction to Boosting“, Journal of Japanese Society for Artificial Intelligence, Vol. 14, No. 5, 1999, pp. 771-780

[3] Bradski, G. \& Kaehler A. (2008). Learning OpenCV: Computer Vision with the OpenCV Library, ORielly Media, 978-0-59651613-0, Sebastopol (USA)

[4] Green, D. (2011). The Future of Autonomous Ground Logistics. Convoys in Department of Defense, School of Advanced Military Studies - United Stadt Army Command and General Staff Collage, USA

[5] Olclay, A. \& Hatice, D. (2010). "Using AdaBoost classifier in hierachial framework for classifying images of marble slabs", Expert Systems with Application, Vol. 37, 2010, pp. 8814-8821

[6] Weckler, P., Bowser, T., Wang, N. \& Mathanker S. (2011). „AdaBoost classifiers for pecan defect classification“, Computer and Electronics in Agriculture, Vol. 77, pp. 60-68

[7] Tombari, F, Di Stefano, L. \& Mattocia, S. (2011). "Efficient template matching for multi channel images", Pattern Recognition Letters, Vol. 32, pp. 694-700

[8] Schoenherr, E. (2009). Moving Future Operation with Convoy Active Safety Technologies (CAST), School of Advanced Military Studies - United Stadt Army Command and General Staff Collage, USA

[9] http://docs.opencv.org/doc/tutorials/tutorials.html (2012).opencv dev team, 2012, Accessed on:2012-6-16

[10] Jong-Yun, K. \& Tae-Yong, K. (2009). "Soccer Ball Tracking unsing Dynamic Kalman Filter with Velocity Control", Sixth International Conference on Computer Graphics, Imaging and Visualization, Vol. 6, pp. 367-374

[11] Bertozzi, M.m Broggi, A., Felisa, M. \& Vezzoni, G. (2006). „Low-level Pedestrian Detection by means of Visible and Far Infra-red Tetra-vision“, Intelligent Vehicle Symposium 2006, pp. 231-236

[12] Bansal, M., Wu, S. \& Eledath, J. (2009). "Pedestrian Association and Localisation in Monocular FIR Video Sequences",Conference of Computer Society, pp. 38-45

[13] Wu, S., Bansal, M., Eledath, J. (2009). "Pedestrian Localization by Apperance Matching and Multi-mode Filtering", Intelligent Vehicle Symposium, pp. 172-178

[14] Seal, A., Bhattacharjee, D., Nasipuri, M. \& Basu, D. K. (2011), "Minutiae Based Thermal Face Recognition using Blood Perfusioin Data", 2011 International Conference on Image Information Processing, pp. 1-4 